\title{
Isogeometric design of elastic arches for maximum fundamental frequency
}

\author{
Attila P. Nagy • Mostafa M. Abdalla • Zafer Gürdal
}

Received: 11 February 2010 / Revised: 2 June 2010 / Accepted: 14 July 2010 / Published online: 12 August 2010

(C) The Author(s) 2010. This article is published with open access at Springerlink.com

\begin{abstract}
The isogeometric paradigm is aimed at unifying the geometric and analysis descriptions of engineering problems. This unification is brought about by employing the same basis functions describing the geometry to approximate the physical response. Non-uniform rational B-splines (NURBS) are commonly used for this purpose and are adopted in the present work for the design of elastic arches. Design for optimal shape and stiffness distribution is considered. Manufacturing constraints are imposed on shape and sizing variables. Shape changes are represented by altering spatial location of the control points and the associated weights. Sizing variables, that control the stiffness distribution, are defined at the control points and interpolated using the same spline basis functions. Since analysis, sizing, and shape design share the same underlying description, consistent discrete sensitivities can be easily evaluated analytically, greatly improving the performance of the optimisation process. While sizing should reflect the influence of local stress states, shape design is preferably performed at a global level. Thus, a multilevel approach is utilised, where shape design is carried out at a coarser level. Projecting the shape design sensitivities bridges the gap between the different levels. A variational formulation of essential manufacturing constraints for sizing and shape optimal design is introduced. The design framework is applied to fundamental frequency maximisation problems.
\end{abstract}

Keywords Isogeometric design · NURBS . Continuous sizing . Multilevel shape design . Manufacturing constraints

A. P. Nagy $(\varangle) \cdot$ M. M. Abdalla · Z. Gürdal Aerospace Structures Chair, Delft University of Technology, Kluyverweg 1, 2629 HS Delft, The Netherlands e-mail: a.p.nagy@tudelft.nl

\section{Introduction}

The concept of isogeometric analysis (Hughes et al. 2005) was introduced to alleviate the difficulties and inaccuracies primarily originating from the different geometric representations employed for design and analysis in engineering problems. The isogeometric approach represents a giant leap toward the synthesis between computer-aided design and numerical analysis. This novel branch of computational sciences proposes to discretise a structure using its intrinsic, computer-aided geometric definition. Thus, the exact geometry is encapsulated at the coarsest level, thereby eliminating the need for subsequent communication with the CAD model and simplifying mesh refinement (Cottrell et al. 2007). The isoparametric concept is invoked to approximate the solution. The superiority of the isogeometric framework over standard polynomial-based finite element analysis is argued from numerous perspectives as discussed in Bazilevs et al. (2006) and Evans et al. (2009). Efficient numerical integration schemes for isogeometric analysis are proposed and compared to the Gaussian quadrature rule (Hughes et al. 2010). Isogeometric analysis of structural vibrations is extensively studied in Cottrell et al. (2006) and Hughes et al. (2008). Although the current version of isogeometric analysis relies on a non-uniform rational B-spline (NURBS) based framework, it is interesting to note that the application of more generic geometric forms, e.g. T-splines, is also viable (Bazilevs et al. 2010).

A fundamental difficulty in structural shape optimisation originates from the different geometric descriptions used for shape design and analysis. Geometric approximation inherent in the finite element mesh introduces inaccuracies in the response analysis, and these cannot be diminished by increasing the polynomial order (Szabó et al. 2004). Considering shape optimal design, both the unavoidable 
iteration-wise conversion between computer aided and facet based geometries, and the mesh construction effort (Bazilevs et al. 2010) represent vital sources of inefficiency. Moreover the inherent geometric error is likely to result in inconsistent design sensitivities (van Keulen et al. 2005). Even though retrieving additional information from the underlying geometric model may improve the sensitivities, the existence of a direct link to the CAD database is not always granted in practise (Shen and Yoon 2003). Isogeometric methods possess several essential qualities that can be used to remedy the aforementioned difficulties, making them ideal for shape design.

Isogeometric shape optimisation has been introduced for plane elasticity problems (Cho and Ha 2009; Wall et al. 2008). The application of T-splines for more efficient computation of shape optimal designs has also been recently proposed (Ha et al. 2008). Other works on isogeometric shape design include extensions to heat transfer (Choi and Kwak 2009) and three-dimensional elasticity (Hassani et al. 2009).

Thin-walled structures, typically modelled as Cosserat continua during the design phase, are of particular importance in aerospace and automotive applications. Simultaneous structural sizing and shape optimisation may be easily performed in essence and this extends the feasible design space (Zhou et al. 2004). In a recent paper, the authors proposed an integrated isogeometric sizing and shape optimisation framework for the design of curved structures (Nagy et al. 2010). A distinction is made between the interpolation of stiffness properties and design variables. While design variables are directly interpolated through the spline mapping, a reciprocal interpolation scheme is utilised for the stiffness properties to preserve continuous variation of the stress resultants. Relying on the convex hull property of the shape functions, positive definiteness of the global stiffness matrix is preserved during the optimisation process. Using non-uniform rational B-splines, shape changes are represented by altering the spatial location of control points and the corresponding weights. While sizing should reflect local stress states, shape design is performed preferably at a global level. Thus, a multilevel design approach is proposed, where shape design is carried out at a coarser level. Projecting the shape design sensitivities bridges the gap between the different levels. The formulation is successfully applied to compliance minimisation of elastic arches.

The current work extends the design formulation given in Nagy et al. (2010) to fundamental eigenfrequency maximisation of elastic arches. The framework is extended to account for common production requirements. These are met by imposing constraints on the curvature of the arch and the rate of change of sizing variables. The manufacturing constraints are formulated using a variational approach.
Design of elastic arches for natural frequencies is addressed in numerous works. Initially interest was devoted to structural sizing to prevent vibrational resonance in rotary machinery and in other aeronautical and civil applications. The early work employed a method of successive iterations to solve the boundary value problem and increase the maximum fundamental frequency of straight beams (Niordson 1965; Brach 1968; Karihaloo and Niordson 1973). Design for higher order natural frequencies was also addressed (Olhoff 1976, 1977). Generic formulation of the optimality criterion within a finite element framework is discussed in Kamat and Simitses (1973) and Takewaki (1996). Unlike the latter works, continuous variation of arbitrary sizing parameter along the beam's span is reflected in the analog equation solution (Katsikadelis and Tsiatas 2006). Periodic distribution of sizing variables has been investigated more recently (Gajewski and Piekarski 1994). Exact derivation for shape optimal freely vibrating shallow arches was presented by of Plaut and Olhoff (1983). The work was extended to combined sizing and shape design (Olhoff and Plaut 1983). However, analytical formulations were restricted to simple geometries and the quest to find more generic, robust, and flexible approaches continued. For an extensive survey of structural shape optimisation, the reader is referred to the works of Haftka and Grandhi (1986) and Chang and Choi (1992). More recent reviews can be found in the articles cited hereinafter.

The rest of this paper is organised as follows. A brief overview of non-uniform rational B-splines is provided in Section 2. Isogeometric analysis of structural vibrations is discussed in Section 3. The design framework is reviewed in Section 4. Manufacturing constraints relevant for elastic arches are introduced in Section 5. A generic formulation of the optimality criterion for maximum fundamental frequency is provided in Section 6. The results of the example problems are discussed in Section 7 in greater depth. The work is summarised and some final conclusions are drawn in Section 8.

\section{Geometric preliminaries}

A $d$-dimensional univariate NURBS is a piecewise rational curve composed as the linear combination of rational basis functions of degree $p$ and the associated coefficients, commonly referred to as control points in $\mathbb{R}^{d}$, i.e.

$\mathbf{r}(\xi)=\frac{\sum_{i=1}^{n} N_{i, p}(\xi) w_{i} \mathbf{P}_{i}}{\sum_{i=1}^{n} N_{i, p}(\xi) w_{i}}=\sum_{i=1}^{n} R_{i, p}(\xi) \mathbf{P}_{i}$, 
where $\mathbf{P}_{i}$ are the (non-homogeneous) control points, $w_{i}$ are the associated weights, $N_{i, p}(\xi)$ and $R_{i, p}(\xi)$ represent the non-rational and rational spline basis functions. Setting all weights to unity results in a B-spline. Basically, a NURBS in $\mathbb{R}^{d}$ is the projected image of the corresponding B-spline in $\mathbb{R}^{d+1}$ defined by the control points of homogeneous form $\mathbf{P}_{i}^{w}=\left\{\mathbf{P}_{i} w_{i}, w_{i}\right\}^{T}$.

A knot vector is defined as a non-decreasing sequence of real numbers on a unit interval that contains the co-ordinates in the parametric space of the curve

$\Xi=\left\{\xi_{1}=0, \ldots, \xi_{n+p+1}=1\right\}$

where $\xi_{i}$ and $n$ denote a knot value and the number of basis functions, respectively. Depending on the equal or unequal spacing of the knots, knot vectors may be uniform and non-uniform. The characteristic intervals $\left[\xi_{i}, \xi_{i+1}\right)$ and $\left[\xi_{1}, \xi_{n+p+1}\right]$ are called knot span and patch, respectively. While $p+1$ multiplicity of the first and last knots yields an open knot vector and ensures endpoint interpolation of the curve, multiple interior knots reduce the continuity locally.

The B-spline basis function of degree $p$ is obtained using the recurrence formula

$$
\begin{aligned}
N_{i, 0}(\xi)= & \begin{cases}1 & \text { if } \xi_{i} \leq \xi<\xi_{i+1} \\
0 & \text { otherwise }\end{cases} \\
N_{i, p}(\xi)= & \frac{\xi-\xi_{i}}{\xi_{i+p}-\xi_{i}} N_{i, p-1} \\
& +\frac{\xi_{i+p+1}-\xi}{\xi_{i+p+1}-\xi_{i+1}} N_{i+1, p-1} .
\end{aligned}
$$

The basis functions are non-negative, have compact support and constitute partition of unity at each parametric location. Generally, the set of homogeneous control points and the knot vector fully determine the geometric characteristics of NURBS curves. For a more elaborate discussion, the reader is advised to consult the handbook of Piegl and Tiller (1995) and its references.

Isogeometric analysis and design requires differentiation with respect to parameters defining the curve. The $k$-th derivative of the NURBS represented in (1) with respect to parameter $\xi$ is given by

$$
\frac{\partial^{k} \mathbf{r}(\xi)}{\partial \xi^{k}}=\sum_{i=1}^{n} R_{i, p}^{(k)}(\xi) \mathbf{P}_{i}
$$

where $R_{i, p}^{(k)}(\xi)$ denotes the $k$-th derivative of a rational basis function with respect to the parameter $\xi$, which can be obtained using the Leibniz' rule of successive differentiation, i.e.

$$
\begin{aligned}
R_{i, p}^{(k)}(\xi)= & \frac{\partial^{k} R_{i, p}(\xi)}{\partial \xi^{k}}=\frac{1}{\sum_{j=1}^{n} N_{j, p}(\xi) w_{j}}\left\{N_{i, p}^{(k)}(\xi) w_{i}\right. \\
& \left.-\sum_{l=1}^{k}\left(\begin{array}{l}
k \\
l
\end{array}\right) R_{i, p}^{(l)}(\xi) \sum_{j=1}^{n} N_{j, p}^{(k-l)}(\xi) w_{j}\right\} .
\end{aligned}
$$

The derivatives of the curve with respect to both a control point co-ordinate and $\xi$ can be written in component form as

$$
\frac{\partial^{k}}{\partial \xi^{k}} \frac{\partial r^{q}(\xi)}{\partial P_{i}^{s}}=\left\{\begin{array}{ccc}
R_{i, p}^{(k)}(\xi) & \text { if } & q=s \\
0 & \text { if } & q \neq s
\end{array} .\right.
$$

Derivatives with respect to both the weight $w_{i}$ and $\xi$ can be obtained as

$$
\begin{aligned}
\frac{\partial^{k}}{\partial \xi^{k}} \frac{\partial \mathbf{r}}{\partial w_{i}}=\frac{1}{w_{i}}\{ & {\left[\mathbf{P}_{i}-\mathbf{r}(\xi)\right] R_{i, p}^{(k)}(\xi) } \\
& \left.-\sum_{l=1}^{k}\left(\begin{array}{l}
k \\
l
\end{array}\right) \mathbf{r}^{(l)}(\xi) R_{i, p}^{(k-l)}(\xi)\right\} .
\end{aligned}
$$

\section{Structural vibration of elastic arches}

The elastic strain and kinetic energy functionals of freely vibrating undamped planar Bernoulli-Euler beams with linearly isotropic elastic constitutive law can be written as

$$
\begin{aligned}
& \mathcal{U}(\tilde{\mathbf{u}}, t)=\frac{1}{2} \int\left[E A\left(\lambda-\lambda_{0}\right)^{2}+E I\left(\kappa-\kappa_{0}\right)^{2}\right] d s_{0}, \\
& \mathcal{T}(\dot{\tilde{\mathbf{u}}}, t)=\frac{1}{2} \int \rho A \dot{\tilde{\mathbf{u}}}^{2} d s_{0},
\end{aligned}
$$

where $\tilde{\mathbf{u}}, E, A, I$, and $\rho$ represent the continuous displacement field, the elastic modulus, the cross-sectional area, the second moment of area, and the material density, respectively. The overdot denotes differentiation with respect to time. The terms $\lambda$ and $\kappa$ in (8) denote the stretch and the curvature of the beam's centroid axis in the deformed configurations and can be written as (Simo 1984)

$\lambda=\frac{d s}{d s_{0}}, \quad \kappa=\frac{\mathbf{r}^{\prime} \times \mathbf{r}^{\prime \prime}}{\left\|\mathbf{r}^{\prime}\right\|^{3}} \frac{d s}{d s_{0}}$

with $\mathbf{r}=\mathbf{r}_{0}+\tilde{\mathbf{u}}$. The symbols $\mathbf{r}_{\mathbf{0}}$ and $d s_{0}$ represent a point on the beam's centroid axis and the differential arch length 
in the initial configuration, respectively. No subscript indicates the current configuration. Primes over a symbol denote successive differentiation with respect to $\xi$, the variable of parameterisation. For small deformations, the equation of motion can be derived using Hamilton's principle, which furnishes the general eigenvalue problem of discrete form as

$(\mathbf{K}-\mu \mathbf{M}) \mathbf{u}=0$,

where $\mathbf{K}, \mathbf{M}$, and $\mu$ represent the consistent stiffness and mass matrices, and the square of the frequency of the free vibration, respectively. The mode shape approximating the continuous solution is given by $\tilde{\mathbf{u}} \simeq \mathbf{u}$. Displacements associated with the vibration mode are defined at the control points and the rotationless nature of the formulation is emphasized; for a more thorough discussion the reader is referred to Cottrell et al. (2006), Echter and Bischoff (2009). Isogeometric formulation of stiffness and mass matrices is given as

$\mathbf{K}=\int \mathbf{D}^{T} \mathbf{C D} J_{0} d \xi$,

$\mathbf{M}=\int \rho A \mathbf{R}^{T} \mathbf{R} J_{0} d \xi$,

with

$\mathbf{C}=\left[\begin{array}{cc}E A & 0 \\ 0 & E I\end{array}\right], \quad \mathbf{D}=\left[\begin{array}{cc}\frac{\partial\left(\lambda-\lambda_{0}\right)}{\partial \mathbf{u}} & \frac{\partial\left(\kappa-\kappa_{0}\right)}{\partial \mathbf{u}}\end{array}\right]^{T}$,

$J_{0}=\left\|\mathbf{r}_{0}^{\prime}\right\|, \quad \mathbf{R}=\left[\begin{array}{ccccc}R_{1, p} & 0 & \ldots & R_{n, p} & 0 \\ 0 & R_{1, p} & \ldots & 0 & R_{n, p}\end{array}\right]$

The mode shape is normalised with respect to the symmetric, positive definite mass matrix, i.e.

$\mathbf{u}^{T} \mathbf{M u}=1$.

\section{Isogeometric design optimisation}

\subsection{Design parameterisation}

In this section the fundamental concepts of design parameterisation of elastic arches treated in Nagy et al. (2010) in greater depth will be recapitulated. In essence, all design variables are defined at the control points within the proposed isogeometric framework. Sizing variables comprise the characteristic dimensions of the cross section, e.g. the inner radius of a tubular section or the thickness of a web. Cross-sectional properties in the parametric space are obtained through regular spline interpolation, i.e.

$b(\xi)=\sum_{j=i}^{i+p} b_{j} R_{j, p}(\xi)$,

where $b(\xi), b_{j}$, and $i$ represent an arbitrary sizing variable of a given parametric location, the associated control points, and the NURBS co-ordinate (Cottrell et al. 2009), respectively.

In order to ensure continuous variation of the stress resultants along the arch's span, the stiffness terms are interpolated in a reciprocal sense (Wesseling 2001; Abdalla et al. 2007), which furnishes the following mapping:

$\mathbf{C}(\xi)=\left[\sum_{j=i}^{i+p} \mathbf{C}_{j}^{-1} R_{j, p}(\xi)\right]^{-1}$

where $\mathbf{C}(\xi)$ and $\mathbf{C}_{j}$ represent the stiffness matrix, see (11), of a given parametric location and the associated control points, respectively. The delineated continuous sizing approach relies on the convex hull property of the underlying geometry, thereby ensuring that the global stiffness matrix remains positive definite during the sizing procedure. It is to be emphasized that the reciprocal interpolation scheme of (15) is strictly utilised to compute the stiffness terms and their sensitivities. Other quantities, such as structural volume, are obtained by relying on (14).

Finally, within the NURBS based isogeometric design framework, shape changes may be introduced by altering the spatial location of non-homogeneous control points and the corresponding weights, all of which represent potential shape design variables.

\subsection{Shape change norm}

Contrary to sizing, shape optimisation problems are typically more difficult to tackle. One major concern of structural shape design is to obtain meaningful shapes adequate for both physical analysis and future manufacturing purposes. Thus, the way shape constraints are formulated is crucial for the optimisation point of view. It has been already concluded (Nagy et al. 2010) that a set of linear constraints between adjacent control points, successfully used to solve isogeometric shape design problems of planar or three-dimensional elastic continua (Ha et al. 2008; Hassani et al. 2009), results in ambiguous shapes when applied to design Cosserat media. The following Sobolev semi-norm was introduced to attain better control over the permitted shape variation (Nagy et al. 2010):

$S=\left(\mathbf{p}_{j}-\mathbf{p}\right)^{T} \mathbf{H}\left(\mathbf{p}_{j}-\mathbf{p}\right)$, 
where $\mathbf{H}$ represents the non-dimensionalised linear stiffness matrix in the rotationless displacement formulation. The symbols $\left(\mathbf{p}_{j}-\mathbf{p}\right)$ and $d$ denote the change in control point co-ordinates at the $j$ th iteration and the characteristic dimension of the shape, respectively. In a general sense the characteristic dimension could be the diameter of the sphere containing the geometry constituting single or multiple patches. Limiting the proposed shape change norm induces a single constraint, which bounds the allowed shape variation by naturally incorporating both length and curvature changes of the entire arch. In essence, gradual relaxation of the shape change norm during the optimisation procedure can be viewed as a shape regularisation technique supporting convergence toward the optimal design. In case weights are also included as design variables, the generalized shape measure is applied on the B-spline in $\mathbb{R}^{3}$ with weights considered as additional co-ordinates.

\subsection{Sensitivity analysis}

The isogeometric framework delineated thus far facilitates the evaluation of design sensitivities analytically, which yields consistent sensitivity information of enhanced accuracy (Cho and $\mathrm{Ha} 2009$ ) and reduces computational time, leading to significant performance improvements.

Considering the eigenvalue problem presented in (10) and (13), the derivative of an eigenvalue with respect to an arbitrary design variable $x$ can be obtained as

$\frac{\partial \mu}{\partial x}=\mathbf{u}^{T}\left(\frac{\partial \mathbf{K}}{\partial x}-\mu \frac{\partial \mathbf{M}}{\partial x}\right) \mathbf{u}$.

Notice that the dependence on an arbitrary sizing variable is contained in both $A\left(b_{j}\right)$ and $I\left(b_{j}\right)$. Thus, sizing sensitivities are evaluated by computing the derivatives with respect to $A$ and $I$ and applying the chain rule. Evaluation of general shape sensitivities further involves higher-order differentials of the NURBS curve as derived in Section 2.

\subsection{Multilevel optimisation}

Despite the fact that sizing and shape optimal design can be combined in a straightforward fashion, it is essential to treat the two sets of design variables separately while aiming for an enhanced design strategy. Basically, increasing the number of sizing variables enables more effective distribution of structural mass, affecting the local stress field more accurately with a moderate increase in computational cost. In contrast, enriching the shape design space and performing shape design and analysis at identical levels of refinement may yield shapes that result in high local strain gradients, a generally undesired feature. In order to resolve this inherent difficulty, a multilevel approach of narrowtree form was proposed (Nagy et al. 2010). Consequently, design variables sufficient to alter the shape at a reasonable fidelity are defined at a coarse grid, i.e. upper level, while sizing variables are defined in a more refined lower level model. Physical and sensitivity analyses of higher accuracy are performed at the refined, i.e. lower level, model.

\section{Manufacturing constraints}

Production requirements typically originate from the buildup and characteristics of the employed production device. In the case of elastic arches the designer may impose sizing or shape related constraints. A common constraint is the Niordson constraint (Niordson 1965), i.e. limiting the rate of change of a given sizing variable. In addition, a constraint on the curvature of the initial configuration may be imposed for shape design. These constraints are formulated in this section in a way suitable for implementation within the isogeometric design framework. This is achieved using a new variational formulation of the individual constraints.

\subsection{Niordson constraint}

Limiting the rate of change of an arbitrary sizing variable characterising the cross section was originally suggested by Niordson (Niordson 1965) and has been extensively used for beam type structures (Rozvany 1989). The differential constraint to be satisfied at each point of the domain is formulated as

$-\eta \leqslant \frac{d b}{d s_{0}} \leqslant \eta$

with $\eta$ denoting a predefined constant setting the limit. Niordson constraints may be imposed as

$\left(\frac{d b}{d s_{0}}\right)^{2}-c \leq 0, \quad$ with $c=\eta^{2}$.

The contribution of the constraint to the Lagrangian can be written as (Haftka and Gürdal 1992)

$\mathcal{L}^{b}=\int\left[\left(\frac{d b}{d s_{0}}\right)^{2}-c\right] \zeta d s_{0}$,

where $\zeta$ denotes the Lagrange multiplier function. A finite set of constraints to replace (19) can be obtained by discretising the Lagrangian. To this end, the Lagrange multiplier function $\zeta$ is approximated using control point values and NURBS interpolation. Note that the NURBS interpolation preserves the non-negativity of $\zeta$ over the domain 
once the control point values are restricted to be positive. Employing the summation convention, the discrete form of (20) may be written as

$\mathcal{L}^{b} \approx T_{i j k} b_{i} b_{j} \zeta_{k}-c_{k} \zeta_{k}$,

where the coefficients are given as

$T_{i j k}=\int \frac{1}{J_{0}} R_{i}^{(1)} R_{j}^{(1)} R_{k} d \xi, \quad c_{k}=\int J_{0} R_{k} d \xi$.

A discrete set of constraints is identified as the coefficient of the Lagrange multipliers $\zeta_{k}$. Thus (19) is replaced by the finite set of constraints

$g_{k}^{b}=T_{i j k} b_{i} b_{j}-c_{k} \leq 0$,

with $k=1, \ldots, n$ numbering the control points.

While for pure sizing problems it is sufficient to evaluate and store the multilinear form $T_{i j k}$ and $c_{k}$ prior to the optimisation procedure, note that they are functions of the weights, which should be taken into account for shape optimal design.

\subsection{Curvature constraint}

Although limiting the shape change norm and/or employing a multilevel design scheme may be considered as shape regularisation, both methods are intended to prevent the optimiser from prematurely converging to physically meaningless designs. So far there are no geometric constraints applied on the structure. In this subsection, we propose to formulate a pure geometric constraint to limit the curvature of the arch centreline. The curvature constraints may be formulated as

$|\kappa|-\kappa_{U} \leq 0$,

where $\kappa_{U}$ is an upper limit on the curvature. It is emphasized that (24) is defined over the entire domain, i.e. span of the arch. Proceeding similarly to the previous section, (24) is replaced by the finite set of constraints

$g_{k}^{\kappa}=\int J_{0}\left(\kappa^{2}-\kappa_{U}^{2}\right) R_{k} d \xi$.

Replacing the continuous Niordson or curvature constraint by a finite set of constraints entails local constraint violations. In general, the amplitude of the local constraint violation decreases as the number of control points is increased. The convergence behaviour of the Niordson constraints is much better than that of the curvature constraints, which strongly depend on multilevel design considerations as discussed in Section 7.3.3.

\section{Problem formulation}

Isogeometric design of elastic arches for maximum fundamental frequency is considered. The mathematical formulation of a general optimisation problem can be written as

$$
\begin{array}{cl}
\min _{\mathbf{x}} & \frac{1}{\mu_{0}} \\
\text { subjected to } & V=V_{0} \\
& S \leq S_{0} \\
& g^{b} \leq 0 \\
& g^{\kappa} \leq 0 \\
\text { with } & \mathbf{x}=\{\mathbf{b}, \mathbf{p}, \mathbf{w}\},
\end{array}
$$

where $\mu_{0}, V_{0}$, and $S_{0}$ denote the square of the fundamental eigenfrequency, the initial volume, and the upper bound on the shape change norm, respectively. The symbols $\mathbf{b}$, $\mathbf{p}$, and $\mathbf{w}$ in (26f) represent the design variables, i.e. set of characteristic cross-sectional dimensions, control point co-ordinates, and weights, respectively.

The optimisation problem (26) is solved using the globally convergent version of the method of moving asymptotes (GCMMA) (Svanberg 2002). For eigenvalue optimisation problems, it is common that multiple eigenvalues interact (multimodal designs). Multimodality is addressed using the bound formulation (Olhoff 1989), which is available for the users of the GCMMA algorithm (Svanberg 2007).

Limiting the shape change norm in (26c) is achieved through gradual relaxation of the upper bound $S_{0}$ until the plateau of optimal design is reached. It is to be emphasized that the increment size of the relaxation influences the final results of the optimisation procedure.

The only further discussion of the optimisation process concerns the volume constraint (26b). Nonlinear equality constraints do not lend them to being used for MMA type approximations. Several methods have been proposed to circumvent the difficulty (Bletzinger 1993; Zhang et al. 1996). In this paper, the volume constraint is treated in a simple way that allows the use of the original MMA formulation. For sizing and combined sizing and shape optimisation problems we treat the volume constraint as an inequality constraint with $V_{0}$ serving as an upper bound on the volume. The reason this can be done is that the natural frequency grows as a function of design variables, cross-sectional areas in this case, i.e. $\mathbf{b}=\mathbf{A}$, faster than volume. It has been verified that the constraint is active in all optimisation runs. For pure shape design problems we associate a single sizing variable with all control points corresponding to an arch of uniform cross section. The volume constraint (26b) is used to solve for the cross-sectional area of the arch and explicitly eliminate it from the formulation. The optimiser 
solves a problem that contains only shape design variables and the volume constraint is not included.

\section{Results}

\subsection{General modelling considerations}

Common engineering objects are easily parameterised using NURBS, relying on a low-degree spline basis with knot vectors comprising a single or couple of knot spans and employing few control points. Although they exactly encapsulate the geometry, their direct application for isogeometric analysis requires some additional considerations to obtain appropriate computational geometries. While $C^{0}$ continuity of the curvature, generally preferred for Cosserat type of structures, can be achieved by elevating the spline basis to third degree, in order to describe the structural response accurately, the initial geometry needs to be refined. Proper computational models are obtained by employing the $h$-, $p$-, or $k$-refinement schemes (Cottrell et al. 2007). It is also remarked that while a Dirichlet type of boundary condition is easily satisfied by eliminating the corresponding translational degrees of freedoms, fixing rotations within the current rotationless isogeometric analysis requires the formulation of additional multipoint constraints; for more details see Auricchio et al. (2007) or Nagy et al. (2010).

In order to attain a proper basis of comparison, results obtained at different mesh densities are mapped, i.e. $h$ refined, to the finest mesh considered. Then the designs are re-evaluated, which enables justification of the results in an absolute sense. Consequently, the subscript of $f^{(i)}$, with $i$ denoting the number of knot spans, refers to the level of discretisation at which an arbitrary function $f$ is evaluated. This notation is utilised hereinafter. It is to be emphasized that the mapping procedure of the sizing results is analogous to the sequentially applied knot refinement algorithm by replacing the control point co-ordinates with the corresponding sizing variables.

Employing the commonly used notation, initial and optimal values are denoted with a zero subscript and a star superscript, respectively. Finally, the following convergence criteria are utilised hereinafter:

$$
\begin{aligned}
\left|\bar{z}_{i}-\bar{z}_{i-1}\right| & \leq 10^{-5}, \\
\left|\bar{S}_{i}-\bar{S}_{i-1}\right| & \leq 10^{-4}, \\
\max \left(\left|\overline{\mathbf{x}}_{i}-\overline{\mathbf{x}}_{i-1}\right|\right) & \leq 10^{-3},
\end{aligned}
$$

with $\bar{z}, \bar{S}$, and $\overline{\mathbf{x}}$ denoting the normalised bound parameter, shape change norm, and design variables, respectively. Function values and design variables are normalised using the corresponding values of the initial, nominal design.

\subsection{Sizing of a simply supported beam}

The first problem revisits Niordson's classic example (Niordson 1965), i.e. sizing of a transversely vibrating, simply supported beam of geometrically similar cross section for maximum fundamental frequency. The straight line representing the beam's centroid can be defined by a first degree B-spline, i.e.

$\Xi=\left\{\begin{array}{llll}0 & 0 & 1 & 1\end{array}\right\}$,

$\mathbf{P}_{1}=\left\{\begin{array}{ll}0 & 0\end{array}\right\}^{T}, \quad \mathbf{P}_{2}=\left\{\begin{array}{ll}L & 0\end{array}\right\}^{T}$,

where $L$ represents the span of the beam of initially uniform circular cross section.

Appropriate computational geometry is generated by first order elevating the spline basis functions to $p=3$, then subdividing the single knot span into 160 uniform knot spans. The latter sequence of procedures is commonly referred to as $k$-refinement (Cottrell et al. 2007), which preserves inter-element continuity of the basis functions. Hereinafter $k$-refinement is denoted by $k$ that refers to $p-1$ as the highest order of inter-element continuity. For instance $k=2$ means that the final geometry is described by cubic $(p=k+1=3$ ) spline basis functions after performing $k$-refinement.

The results are summarized in Table 1 and compare well to Katsikadelis' work employing an analog equation solution (Katsikadelis and Tsiatas 2006). Next to the normalised improvement in fundamental frequency, the table also contains the extreme values of the corresponding sizing distribution.

Optimal sizing variables obtained at the control points are mapped to the arch's span according to (14). The normalised variations of optimal cross-sectional area in the different

Table 1 Sizing results of the simply supported beam with Niordson constraint of increasing limit

\begin{tabular}{lllll}
\hline$c\left(\eta^{\mathrm{a}}\right)$ & $\frac{\omega^{* \mathrm{a}}}{\omega_{0}}$ & $\frac{\omega^{*(160)}}{\omega_{0}^{(160)}}$ & $\frac{A_{\min }^{*}}{A_{0}}$ & $\frac{A_{\max }^{*}}{A_{0}}$ \\
\hline 0 & n.a. & 1 & 1 & 1 \\
0.01 & n.a. & 1.0311 & 0.8042 & 1.1795 \\
$0.04(0.2)$ & 1.049 & 1.0471 & 0.6298 & 1.2383 \\
$0.09(0.3)$ & 1.058 & 1.0554 & 0.4853 & 1.2680 \\
$0.16(0.4)$ & 1.063 & 1.0599 & 0.3868 & 1.2770 \\
$0.25(0.5)$ & 1.066 & 1.0624 & 0.2950 & 1.2830 \\
$9^{\mathrm{b}}$ & n.a. & 1.0654 & 0.0502 & 1.2845 \\
Niordson & 1.066 & n.a. & 0 & 1.2786
\end{tabular}

${ }^{\mathrm{a}} \eta \leqslant d A / d x \leqslant \eta$ see Katsikadelis and Tsiatas (2006) and noting that $c=\eta^{2}$

${ }^{\mathrm{b}}$ Constrained problem with inactive Niordson constraint equivalent to unconstrained design 


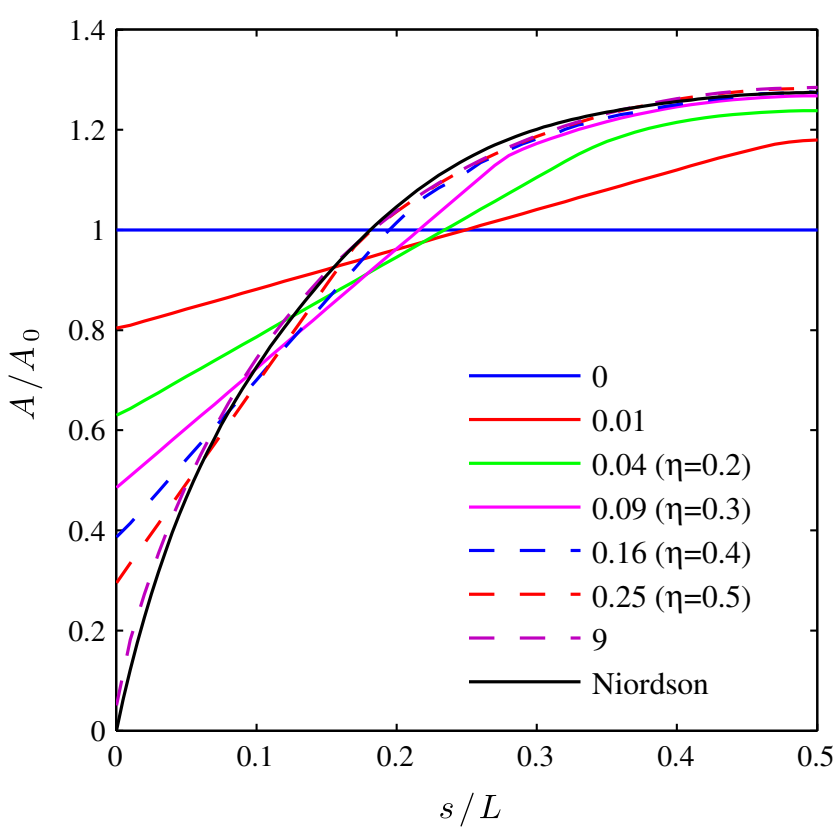

Fig. 1 Normalised cross-sectional area distributions along the beam span with increasing limit of the Niordson constraint

cases are illustrated in Fig. 1. The fundamental mode shape associated with the sized beam is sketched in Fig. 2.

Observe that the constraint formulation naturally incorporates the initial structure of uniform cross-sectional area, i.e. $c=0$. Steeper slopes over reduced arch lengths in the cross-sectional area distribution chart indicate the range of active constraints as the limiting parameter increases and convergence toward the unconstrained design is attained. Setting the limit sufficiently high, e.g. $c=9$ in the current example, yields identical results with the unconstrained design that nicely correlate with Niordson's analytical solution. Note however that the exact solution is singular at the supports, which can not be represented with a class of finite dimensional approximation functions defined in the Sobolev $H^{2}$ space (Evans et al. 2009; Cottrell et al. 2009).

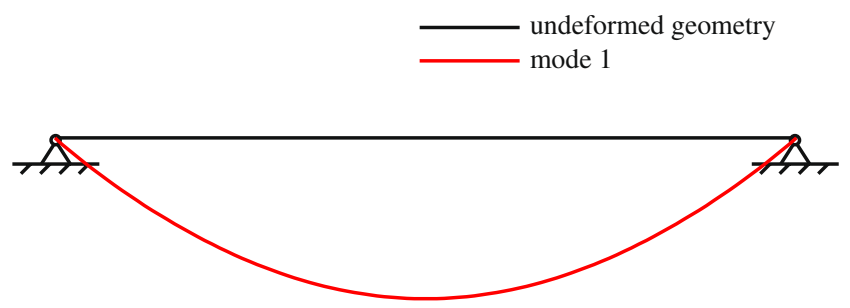

Fig. 2 Fundamental mode shape of the sized beam ( $c=9$, mode shape scaling factor: $10^{-2}$ )

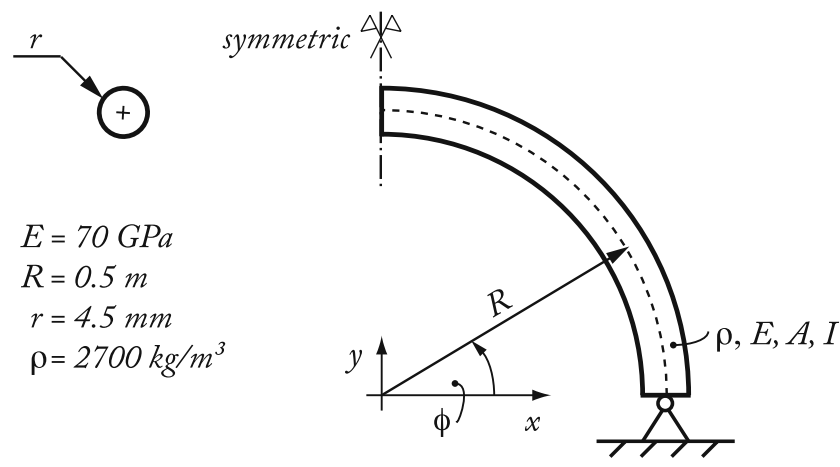

Fig. 3 Schematic representation of the semi-circular arch

\subsection{Isogeometric design of a semi-circular arch}

\subsubsection{Problem statement}

In the second example, isogeometric design of a semicircular arch is studied in a greater depth. The arch of initially uniform, circular cross section of radius $r$ is hinged at both ends as sketched in Fig. 3. The parametric form that encapsulates the exact geometry at the coarsest level utilises a single knot span and four homogeneous control points, i.e.

$\Xi=\left\{\begin{array}{llllllll}0 & 0 & 0 & 0 & 1 & 1 & 1 & 1\end{array}\right\}$,

$\mathbf{P}_{1}^{w}=\left\{\begin{array}{lll}R & 0 & 1\end{array}\right\}^{T}, \quad \mathbf{P}_{2}^{w}=\left\{\begin{array}{lll}R / 3 & 2 R / 3 & 1 / 3\end{array}\right\}^{T}$,

$\mathbf{P}_{3}^{w}=\left\{\begin{array}{lll}-R / 3 & 2 R / 3 & 1 / 3\end{array}\right\}^{T}, \quad \mathbf{P}_{4}^{w}=\left\{\begin{array}{lll}-R & 0 & 1\end{array}\right\}^{T}$,

where $R$ denotes the radius of the circular segment. Essential dimensions and material properties are given in Fig. 3.

\subsubsection{Sizing}

Sizing of the arch was performed at different mesh densities. Therefore the initial geometry was $h$-refined such that the original knot span was subdivided into $5 \cdot 2^{n}$ uniform knot

Table 2 Sizing results of the semi-circular arch with $k=2$, if others not indicated

\begin{tabular}{lccc}
\hline Number of knot spans & $\frac{w^{*(160)}}{w_{0}^{(160)}}$ & $\frac{A_{\min }}{A_{0}}$ & $\frac{A_{\max }}{A_{0}}$ \\
\hline 10 & 1.0938 & 0.4257 & 1.3875 \\
20 & 1.1203 & 0.2603 & 1.2963 \\
40 & 1.1264 & 0.1637 & 1.3317 \\
80 & 1.1288 & 0.1037 & 1.3467 \\
160 & 1.1288 & 0.0655 & 1.3521 \\
$160(k=3)$ & 1.1283 & 0.0579 & 1.3520 \\
$160(k=4)$ & 1.1279 & 0.0520 & 1.3511 \\
$160(k=5)$ & 1.1275 & 0.0487 & 1.3506 \\
\hline
\end{tabular}




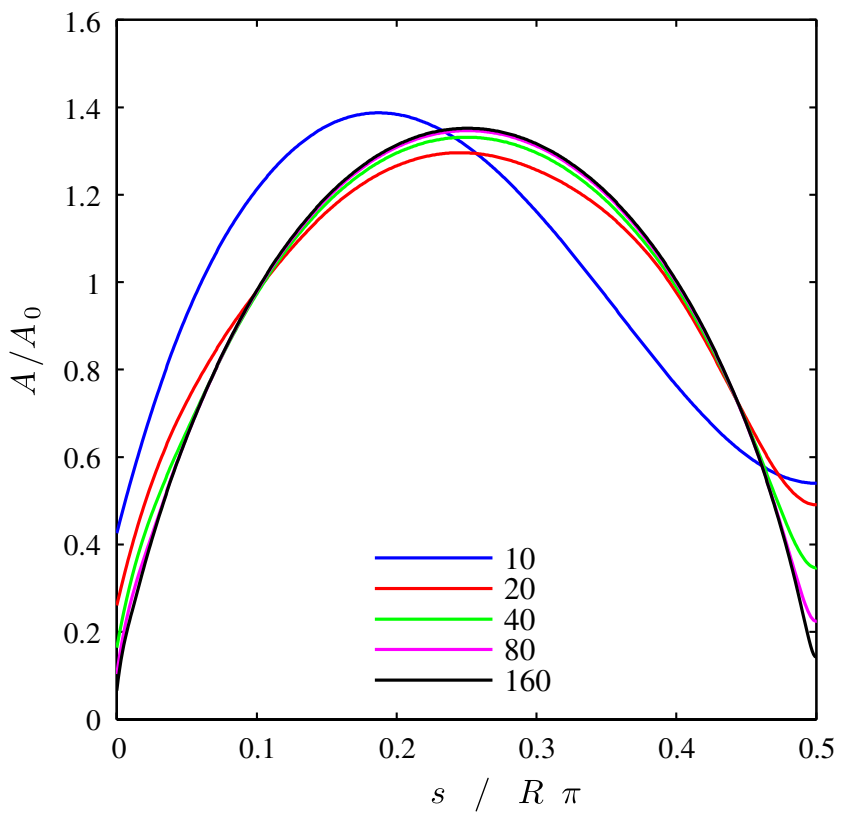

Fig. 4 Normalised cross-sectional area distribution of the sized arch at different mesh densities $(p=3)$

spans $(n=1,2, \ldots, 5)$. Initially no restriction was made on the tapering of the arch, i.e. the Niordson constraint was suppressed from the problem formulation.

The numerical results are summarized in Table 2. Pure sizing design improves the objective function by almost $13 \%$. Normalised cross-sectional area distributions are depicted in Fig. 4. It is interesting to recognise that convergence of the cross-sectional area distributions happens at a slower rate compared to the improvement in the fundamental frequency. The fundamental mode shape of the sized arch is shown in Fig. 5.

The influence of the order of spline basis functions on the optimal sizing parameter distribution was also investigated. The purpose was to study the sensitivity of the final designs to the choice of the order of inter-element continuity, i.e. $k$, of the initial geometry, which is usually given by the

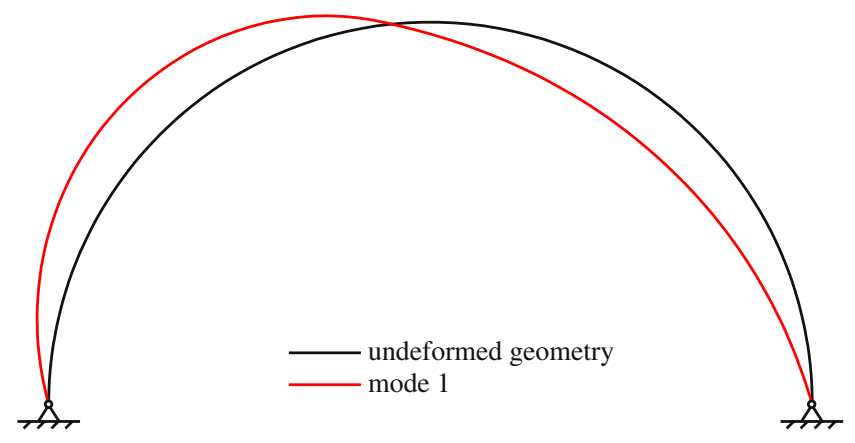

Fig. 5 Fundamental mode shape of the sized arch using 160 knot spans $\left(p=3\right.$, mode shape scaling factor: $\left.10^{3}\right)$
Table 3 Sizing results with added Niordson constraint of increasing limit using 160 knot spans $(k=2)$

\begin{tabular}{llll}
\hline$\tilde{c}^{\mathrm{a}}$ & $\frac{w^{*(160)}}{w_{0}^{(160)}}$ & $\frac{A_{\min }}{A_{0}}$ & $\frac{A_{\max }}{A_{0}}$ \\
\hline 2.5 & 1.0592 & 0.7384 & 1.1821 \\
5 & 1.0784 & 0.6367 & 1.2714 \\
10 & 1.0973 & 0.5082 & 1.3224 \\
25 & 1.1166 & 0.3235 & 1.3452 \\
50 & 1.1246 & 0.2053 & 1.3528 \\
100 & 1.1277 & 0.1450 & 1.3533 \\
\hline
\end{tabular}

${ }^{\mathrm{a}} c=\tilde{c} \cdot A_{0}^{2} /(R \pi)^{2}$

designer and can depend on the particular method used for the construction of the CAD model. Thus, the original spline basis functions were order elevated before the single knot span was subdivided into 160 uniform knot spans. It is to be emphasized however, that elevating the order by one within the $k$-refinement scheme adds only a single control point regardless of the number of knot spans. This means that the total number of unknowns is left essentially the same. The results, listed in the last three rows of Table 2, indicate that the order of inter-element continuity does not have a significant influence on the optimal sizing parameters.

Although several recent works in the isogeometric literature have proven $k$-convergence numerically for regular problems, see references (Cottrell et al. 2006; Hughes et al.

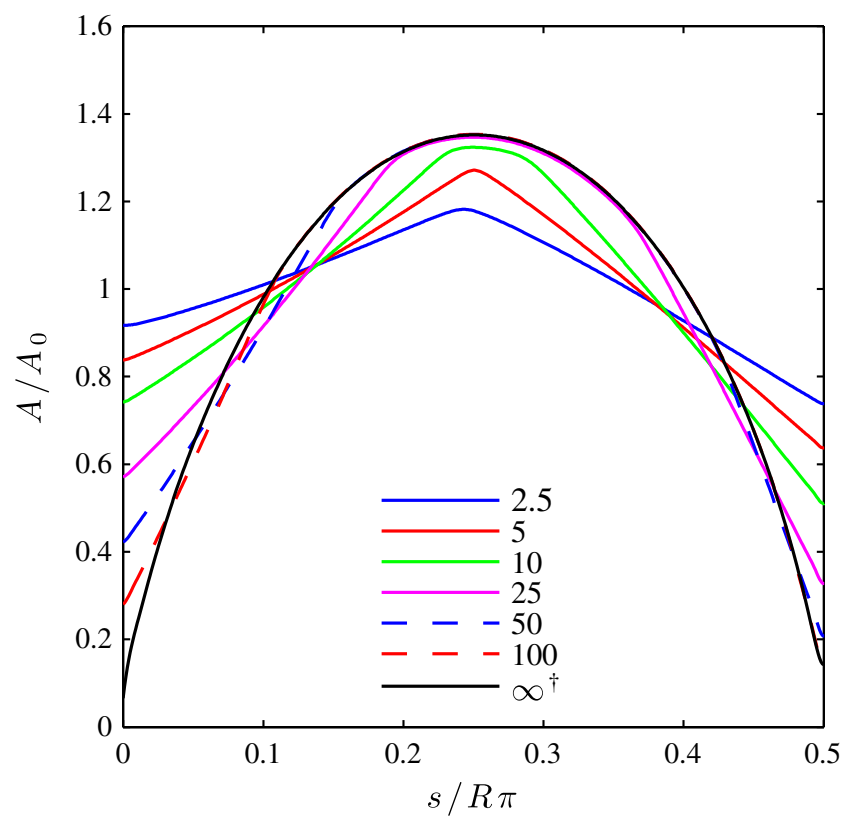

Fig. 6 Normalised cross-sectional area distribution of the sized arch including Niordson constraints at 160 knot spans ( $p=3$; ‘unconstrained design) 
Table 4 Single level shape design results $(k=2)$

\begin{tabular}{|c|c|c|c|c|c|c|}
\hline No. & $\begin{array}{l}\text { Number of } \\
\text { knot spans }\end{array}$ & $\begin{array}{l}\text { Design } \\
\text { variables }\end{array}$ & $d S_{0}$ & $\frac{w^{*(160)}}{w_{0}^{(160)}}$ & $\frac{A^{*}}{A_{0}}$ & $S^{*}$ \\
\hline 1 & 10 & $\mathbf{p}$ & 0.001 & 1.9755 & 1.2182 & 0.5560 \\
\hline 2 & 10 & $\mathbf{p}$ & 0.025 & 1.5865 & 1.1461 & 1.2998 \\
\hline 3 & 10 & $\mathbf{p}, \mathbf{w}$ & 0.001 & 2.3378 & 1.2702 & 0.5070 \\
\hline 4 & 10 & $\mathbf{p}, \mathbf{w}$ & 0.025 & 1.6438 & 1.1558 & 1.2497 \\
\hline 5 & 20 & $\mathbf{p}$ & 0.001 & 2.1086 & 1.2419 & 0.6659 \\
\hline 6 & 20 & $\mathbf{p}$ & 0.025 & 1.9449 & 1.2036 & 2.7139 \\
\hline 7 & 20 & $\mathbf{p}, \mathbf{w}$ & 0.001 & 2.3680 & 1.2743 & 0.5259 \\
\hline 8 & 20 & $\mathbf{p}, \mathbf{w}$ & 0.025 & 2.0948 & 1.2295 & 2.1445 \\
\hline
\end{tabular}

2008; Bazilevs et al. 2007), the results in Table 2 indicate poor $k$-convergence. The reason for this is primarily associated with the uniform partitioning of the knot vector. Placing the interior knots equispaced does not optimally approximate the solution where high gradients occur. Employing non-uniform knot vectors of optimally placed interior knots would possibly improve the convergence, however optimal locations, dependent on the order of the spline basis, are generally hard to obtain (Evans et al. 2009). Similar findings are reported in the authors' previous work (Nagy et al. 2010).

The outcome of the reformulated optimality criterion comprising the Niordson constraint is given in Table 3 . The results were obtained at the finest mesh consisting 160 knot spans. Increasing the limiting parameter shows convergence toward the unconstrained design. The corresponding normalised sizing variable distributions are depicted in Fig. 6.

\subsubsection{Shape optimal design}

Shape design and structural analysis were performed at identical and different levels of discretisation, i.e. both single and multilevel schemes were applied, respectively. The adapted computational models discretising the arch with 10 ,

Table 5 Multilevel shape design results $(k=2)$

\begin{tabular}{lllllll}
\hline No. & $\begin{array}{l}\text { Number of knot spans } \\
\text { shape design/analysis }\end{array}$ & $\begin{array}{l}\text { Design } \\
\text { variables }\end{array}$ & $\frac{w^{*(160)}}{w_{0}^{(160)}}$ & & $A^{*}$ & \\
$A_{0}$ & $S^{*}$ & \\
\hline 1 & $10 / 20$ & $\mathbf{p}$ & 3.0854 & 1.3674 & 0.7499 \\
2 & $10 / 20$ & $\mathbf{p}, \mathbf{w}$ & 3.2473 & 1.3891 & 0.6998 \\
3 & $10 / 40$ & $\mathbf{p}$ & 3.3954 & 1.3999 & 0.9499 \\
4 & $10 / 40$ & $\mathbf{p}, \mathbf{w}$ & 3.3892 & 1.4043 & 1.2498 \\
\hline
\end{tabular}

20, and 40 uniform knot spans were attained through $h$ refinement. A study on the influence of the shape change norm relaxation increment size, i.e.

$d S_{0}=S_{0}^{i+1}-S_{0}^{i}$

was also conducted. However, if otherwise not stated, the limit on the shape change norm was gradually increased with 0.025 increments.

Initially a reduced formulation neglecting the constraint on the arch's curvature was considered. The results presented in Tables 4 and 5 show a profound increase in the fundamental eigenfrequency compared to the sized only designs. The tables contain the normalised cross-sectional

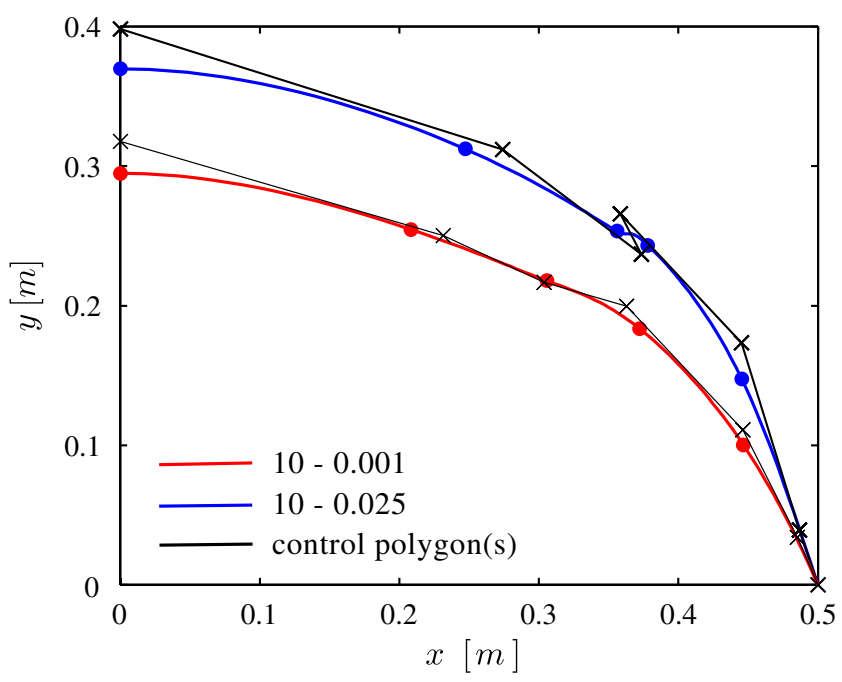

(a)

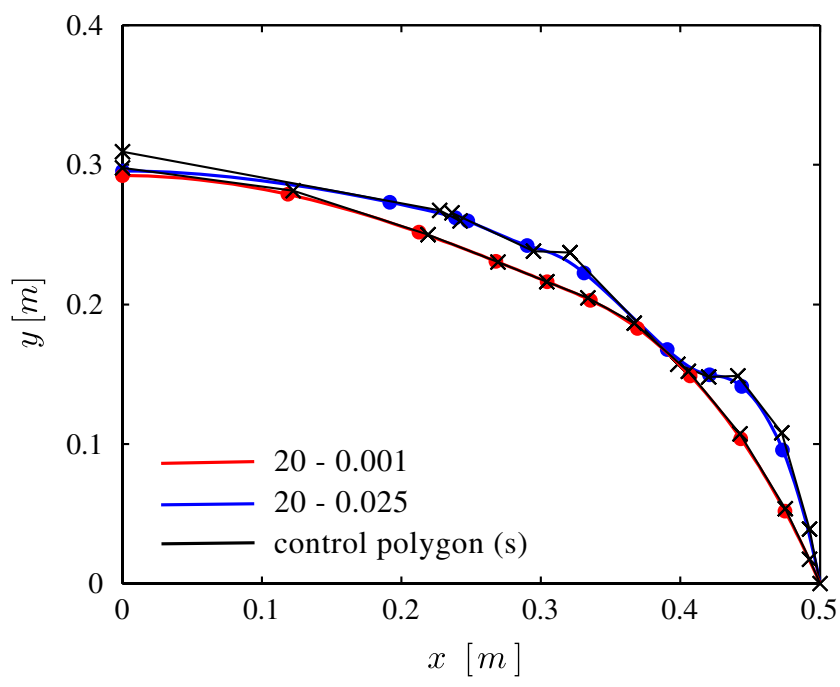

(b)

Fig. 7 Single level shape optimal designs using 10 (a) and 20 (b) knot spans with different shape change norm relaxation increments 


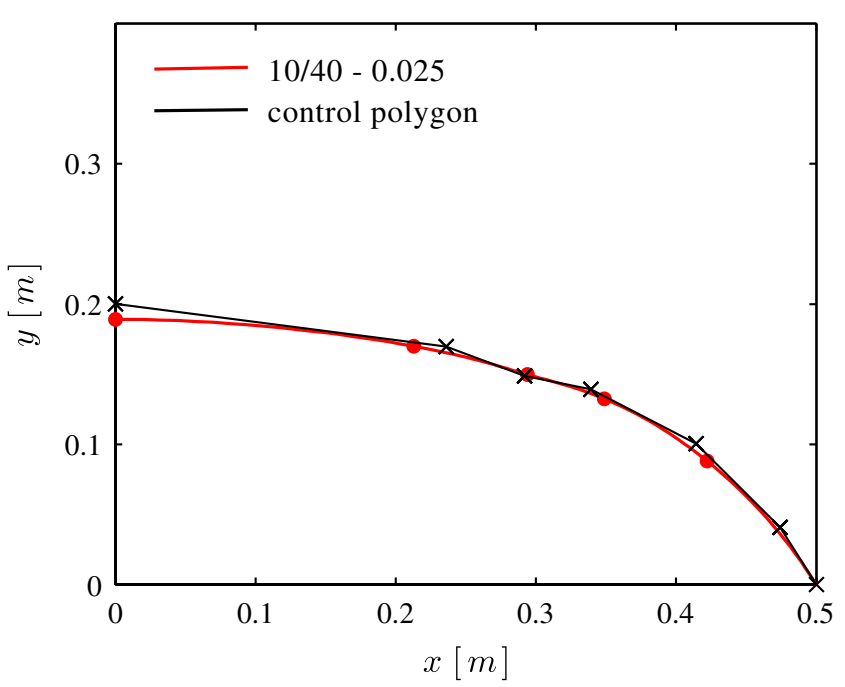

Fig. 8 Multilevel shape optimal design using 10 and 40 knot spans for shape design and analysis, respectively

areas, which keep the volume constraint active during the shape design process. This measure may be interpreted as a scaling factor inversely proportional to the change of corresponding arch length.

In essence the NURBS based geometric description can be fully exploited by employing both control point coordinates and weights as shape design variables. Weights are concluded to be beneficial as shape design is performed at a relatively coarse mesh. Extending the set of shape vari-

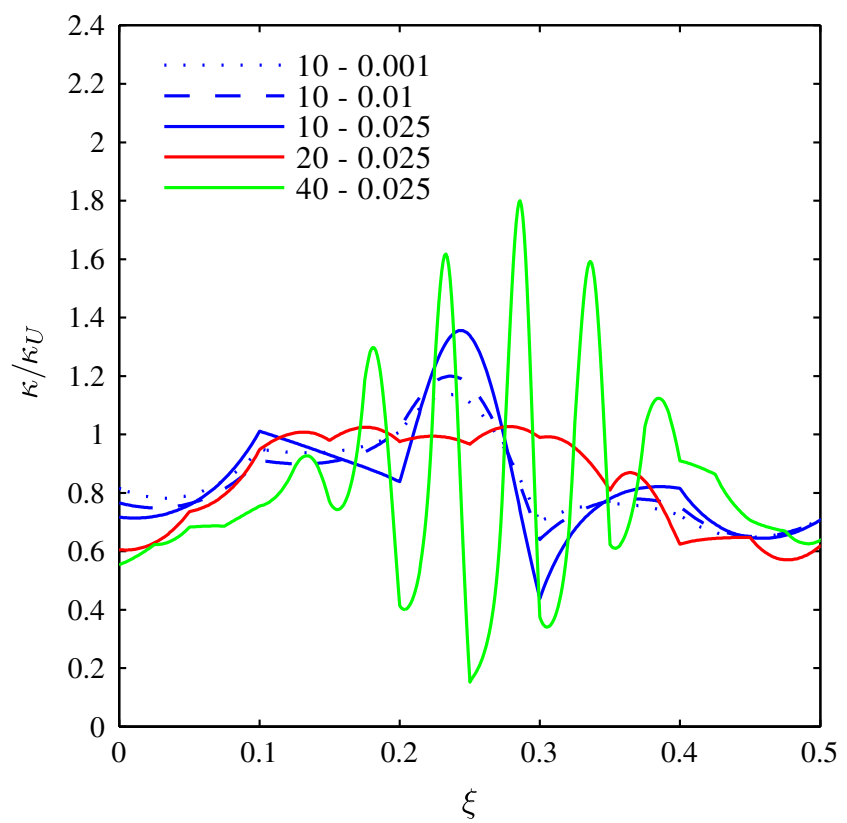

Fig. 9 Curvature constraints satisfaction within the single level design scheme ables, however, does not necessarily lead to better designs, since the optimiser may settle to a worse local optimum, e.g. note designs 3 and 4 in Table 5.

Optimal shapes associated with designs 3, 4 and 7, 8 in Table 4 are depicted in Fig. 7 a and b, respectively. Since the optimal shapes are symmetric with respect to the $y$ axis, for

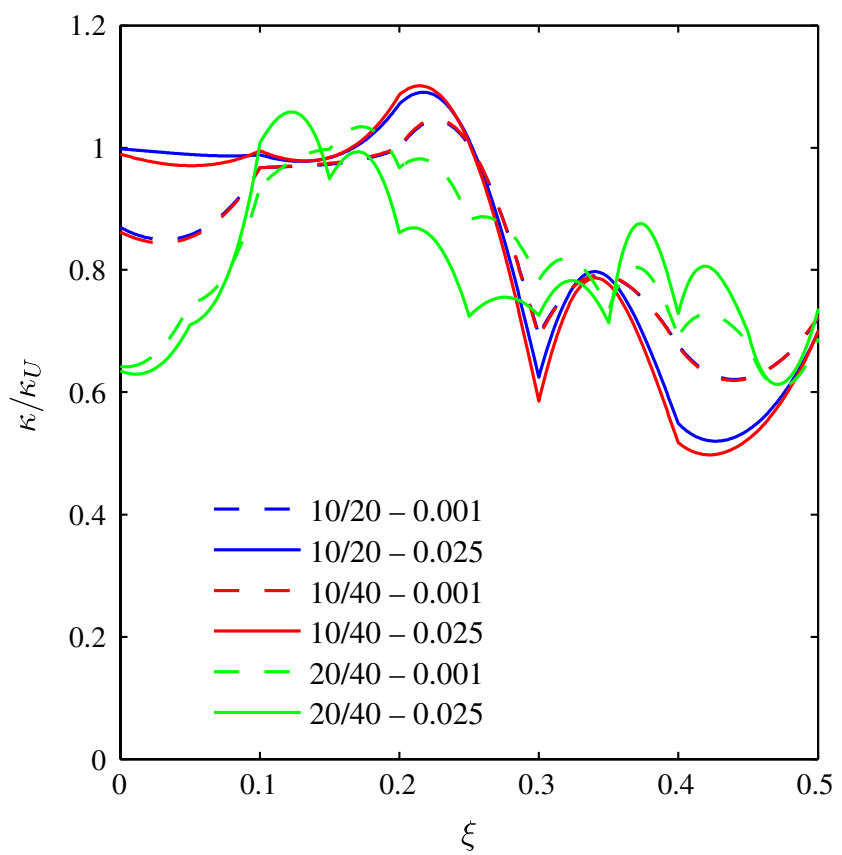

(a)

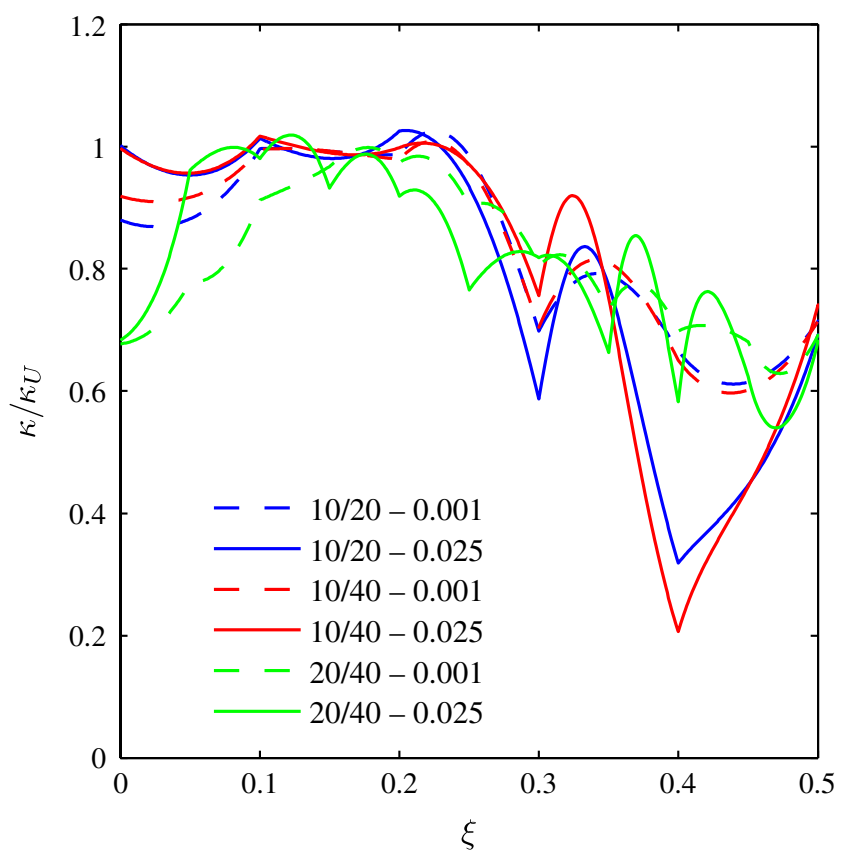

(b)

Fig. 10 Curvature constraints defined on the upper (a) and lower (b) level models within the multilevel design scheme 
Table 6 Results of single level shape design with imposed curvature constraint $(k=2)$

Table 7 Results of multilevel shape design with curvature constraint defined on the upper level model $(k=2)$

Table 8 Results of multilevel shape design with curvature constraint defined on the lower level model $(k=2)$

\begin{tabular}{lllllllll}
\hline No. & $\begin{array}{l}\text { Number of } \\
\text { knot spans }\end{array}$ & $d S_{0}$ & $\frac{w^{*(160)}}{w_{0}^{(160)}}$ & $\frac{A^{*}}{A_{0}}$ & $\epsilon_{1}$ & $\epsilon_{2}$ & $\epsilon_{3}$ & $S^{*}$ \\
\hline 1 & 10 & 0.001 & 1.5701 & 1.1406 & 0.1369 & 0.1439 & 0.0197 & 0.1340 \\
2 & 10 & 0.01 & 1.4847 & 1.1232 & 0.1992 & 0.1518 & 0.0302 & 0.1399 \\
3 & 10 & 0.025 & 1.5474 & 1.1354 & 0.3557 & 0.1479 & 0.0526 & 0.2999 \\
4 & 20 & 0.025 & 1.5049 & 1.1302 & 0.0268 & 0.1878 & 0.0050 & 0.2999 \\
5 & 40 & 0.025 & 1.1533 & 1.0434 & 0.7998 & 0.2038 & 0.1630 & 0.2748 \\
\hline
\end{tabular}

\begin{tabular}{lllllllll}
\hline No. & $\begin{array}{l}\text { Number of knot spans } \\
\text { shape design/analysis }\end{array}$ & $d S_{0}$ & $\frac{w^{*(160)}}{w_{0}^{(160)}}$ & $\frac{A^{*}}{A_{0}}$ & $\epsilon_{1}$ & $\epsilon_{2}$ & $\epsilon_{3}$ & $S^{*}$ \\
\hline 1 & $10 / 20$ & 0.001 & 1.7518 & 1.1749 & 0.0442 & 0.1019 & 0.0045 & 0.1810 \\
2 & $10 / 20$ & 0.025 & 2.2305 & 1.2555 & 0.0907 & 0.1698 & 0.0154 & 0.3499 \\
3 & $10 / 40$ & 0.001 & 1.7555 & 1.1756 & 0.0466 & 0.1039 & 0.0048 & 0.1840 \\
4 & $10 / 40$ & 0.025 & 2.3695 & 1.2758 & 0.1012 & 0.1718 & 0.0174 & 0.3999 \\
5 & $20 / 40$ & 0.001 & 1.6584 & 1.1574 & 0.0343 & 0.0839 & 0.0029 & 0.3229 \\
6 & $20 / 40$ & 0.025 & 1.7305 & 1.1687 & 0.0585 & 0.0879 & 0.0051 & 0.5499 \\
\hline
\end{tabular}

\begin{tabular}{lllllllll}
\hline No. & $\begin{array}{l}\text { Number of knot spans } \\
\text { shape design/analysis }\end{array}$ & $d S_{0}$ & $\frac{w^{*(160)}}{w_{0}^{(160)}}$ & $\frac{A^{*}}{A_{0}}$ & $\epsilon_{1}$ & $\epsilon_{2}$ & $\epsilon_{3}$ & $S^{*}$ \\
& $10 / 20$ & 0.001 & 1.8163 & 1.1866 & 0.0246 & 0.0779 & 0.0019 & 0.1969 \\
1 & $10 / 20$ & 0.025 & 2.9357 & 1.3497 & 0.0262 & 0.1299 & 0.0034 & 0.6499 \\
3 & $10 / 40$ & 0.001 & 1.8603 & 1.1945 & 0.0085 & 0.0480 & 0.0004 & 0.2139 \\
4 & $10 / 40$ & 0.025 & 2.9052 & 1.3465 & 0.0172 & 0.1259 & 0.0022 & 0.6750 \\
5 & $20 / 40$ & 0.001 & 1.6604 & 1.1574 & 0 & 0 & 0 & 0.2320 \\
6 & $20 / 40$ & 0.025 & 2.0228 & 1.2220 & 0.0187 & 0.0559 & 0.0010 & 0.7999 \\
\hline
\end{tabular}


Table 9 Results of multilevel simultaneous sizing and shape design $(k=2)$

\begin{tabular}{lllllll}
\hline No. & $\begin{array}{l}\text { Number of knot spans } \\
\text { shape design/sizing and analysis }\end{array}$ & $\begin{array}{l}\text { Design } \\
\text { variables }\end{array}$ & $\frac{w^{*(160)}}{w_{0}^{(160)}}$ & $\frac{A_{\min }^{*}}{A_{0}}$ & $\frac{A_{\max }^{*}}{A_{0}}$ & $S^{*}$ \\
\hline 1 & $10 / 20$ & $\mathbf{A}, \mathbf{p}$ & 3.3426 & 0.4393 & 1.7460 & 0.7499 \\
2 & $10 / 20$ & $\mathbf{A}, \mathbf{p}, \mathbf{w}$ & 3.5115 & 0.4905 & 1.7694 & 0.6998 \\
3 & $10 / 40$ & $\mathbf{A}, \mathbf{p}$ & 3.6855 & 0.2839 & 1.8098 & 0.9500 \\
4 & $10 / 40$ & $\mathbf{A}, \mathbf{p}, \mathbf{w}$ & 3.6857 & 0.3476 & 1.8174 & 1.2499 \\
\hline
\end{tabular}

better visibility only one half of the arch has been plotted. Note the emergence of distorted control polygons and the clustering of control points accounted for severe localised shape corrugation when utilising the single level scheme. One may easily foresee the evolution of self-intersecting shapes inappropriate for physical analysis. Reducing the increment size of the shape change norm relaxation to a sufficiently small value alleviates the problem but also increases computational time. In contrast, designs attained through the multilevel approach not only result in higher fundamental frequencies, but also inhibit local wave formations and the occurrence of other small shape distortions. As an example, the optimal arch associated with the fourth design of Table 5 is depicted in Fig. 8. The corresponding fundamental mode shape is depicted in Fig. 12.

Now we include the additional curvature constraint to the formulation and set the maximum allowed curvature to $\kappa_{U}=1.25 \cdot \kappa_{0}$, with $\kappa_{0}$ denoting the initial curvature of the arch. The set of design variables was restricted to control point co-ordinates only. The primary goal was to investigate how to enforce the limit within the single and multilevel schemes.

First recall that the curvature constraint in (25) was imposed in a variational sense. Thus, local constraint violations may occur that need to be quantified to distinguish the different solutions. Consequently, three error measures were introduced. The normalised curvature violation indicates the largest local inaccuracy along the entire span of the optimal shape. The normalised arch length along which the constraint is exceeded yields a more global norm. Finally, the product of the latter error measures quantifies the overall constraint satisfaction. The following notation is introduced and utilised hereinafter:

$$
\begin{aligned}
\epsilon_{1} & =\frac{\max [\kappa(\xi)]}{\kappa_{U}} \\
\epsilon_{2} & =\frac{s\left(\xi \mid \kappa(\xi)>\kappa_{U}\right)}{s}, \\
\epsilon_{3} & =\epsilon_{1} \cdot \epsilon_{2}
\end{aligned}
$$

where $\epsilon_{i}$ is the $i$ th error norm and $s$ denotes the arch length. Normalised curvature distribution attained via the single and multilevel design procedures are depicted in Figs. 9 and 10, respectively. Note the different scaling of the figures. Detailed results are presented in Tables 6, 7, and 8.

Reducing the increment size of the shape change norm relaxation is concluded to be beneficial within both design schemes in general. Although all presented results comply with the constraints, results of the different schemes show entirely different trends. Refining the geometry, and hence increasing the number of design variables within the single level scheme, may yield localised constraint violation with increasing amplitudes of error, as illustrated in Fig. 9. The quality of the results is extremely prone to being influenced by the selected increment size.

In contrast, separating the levels of shape design and analysis improves constraint satisfaction without the occurrence of spurious oscillations, as shown in Fig. 10. Note,

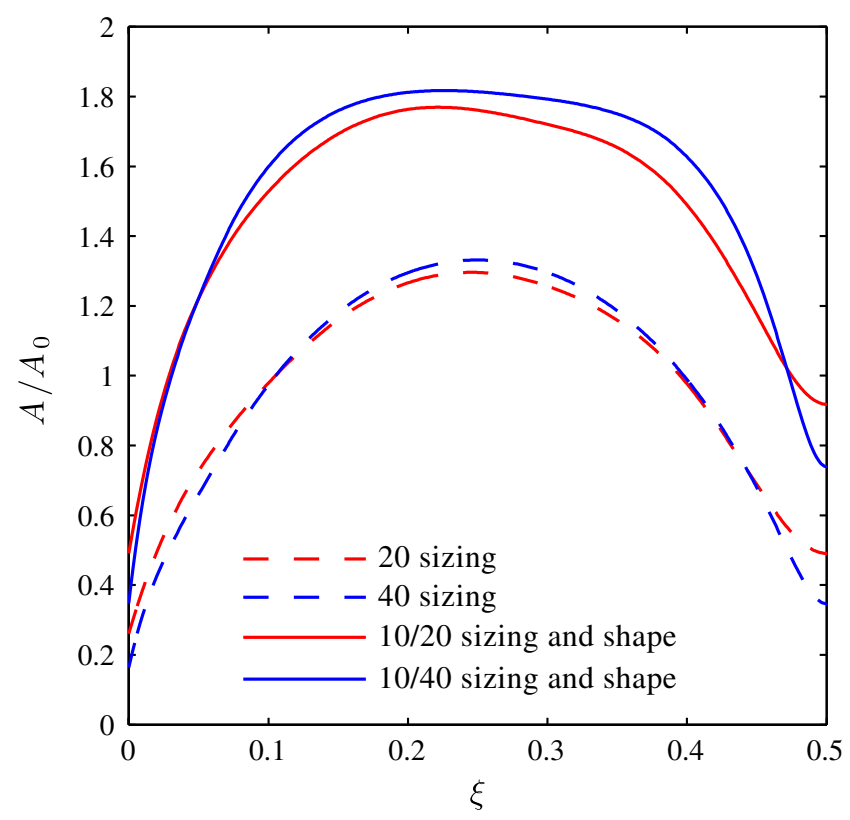

Fig. 11 Normalised cross-sectional area distributions using different multilevel schemes for combined sizing and shape design 
however, that limiting the curvature on the lower level model yields better results, which are generally less sensitive to the size of the shape change norm relaxation increment. This is simply related to the improved quality of constraint approximation, i.e. the constraint is enforced at an increased number of control points. Assuming sufficiently slow relaxation of the shape change norm, it is conjectured that the constraint is satisfied over the entire span of the arch in the limit of successive $h$-refinements of the lower level model.

\subsubsection{Combined sizing and shape optimal design}

Finally, simultaneous sizing and shape optimal design was conducted employing the multilevel scheme proposed in Section 4.4. Recall that shape and sizing variables are defined at the upper and lower level models, i.e. at the coarse and fine grids, respectively. No manufacturing constraints were introduced for convenience of modeling. Initial design values were given using the previously obtained solutions instead of the nominal design. Hence, while shape variables were initialized to shape optimal designs, sizing variables were set equal to the values of the merely sized optimal shapes. The numerical results presented in Table 9 are directly comparable with values of the corresponding shape optimal designs in Table 5. Comparing the solutions, it can be observed that an additional 25-30\% improvement in fundamental frequencies was reached while the shapes remained practically unchanged, i.e. the achieved performance gain was merely related to sizing.

The distribution of normalised cross-sectional areas of designs 2 and 4 are plotted in Fig. 11. As a reference, the figure also contains the distributions associated with the comparable sized only designs. In contrast to the sized only designs, the integral of the normalised area distributions of both sized and shaped arches is not unity. This is due to the fact that the same amount of material has to be distributed along shapes of reduced arch length. Notice how the material related to structural mass and stiffness has been redistributed once shape changes became viable. The fundamental mode shape corresponding to the design 4 is depicted in Fig. 12.

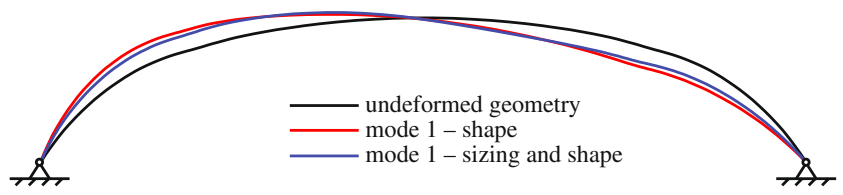

Fig. 12 Fundamental mode shapes of the optimal arches using 10/40 knot spans in the multilevel scheme (mode shape scaling factor: $10^{-2}$ )

\section{Conclusions}

The NURBS based isogeometric design framework has been extended to optimise elastic arches for maximum fundamental frequency. The framework has several distinct features worth highlighting. A distinction has been made between the interpolation of stiffness properties and design variables characterising the arch cross section. Both control point coordinates and the weights have been employed as shape design variables. A shape change norm has been introduced that naturally incorporates both length and curvature changes of the entire arch to attain better control over the permitted shape variation. Realistic shape designs are obtained by gradually relaxing the shape change norm. A multilevel optimisation scheme has been utilised to reflect the distinct nature of sizing and shape design where shape variables are defined on a coarser mesh than sizing variables.

Apart from extending the framework in a structural vibration context, the authors proposed a novel variational formulation of continuous constraints. The new variational formulation was applied to two types of manufacturing requirements: limiting the rate of change of sizing variables and limiting the curvature of the arch centreline. The weak form of the constraints is a pure isogeometric construct that fits seamlessly with the underlying analysis environment. It was found that for shape design it is better to impose the constraints at the finer level within the multilevel scheme to minimise constraint violations.

Acknowledgments The authors are grateful to Professor Krister Svanberg from KTH-Royal Institute of Technology in Stockholm, Sweden for allowing us to use his implementation of the globally convergent method of moving asymptotes.

Open Access This article is distributed under the terms of the Creative Commons Attribution Noncommercial License which permits any noncommercial use, distribution, and reproduction in any medium, provided the original author(s) and source are credited.

\section{References}

Abdalla MM, Setoodeh S, Gürdal Z (2007) Design of variable stiffness composite panels for maximum fundamental frequency using lamination parameters. Compos Struct 81(2):283-291

Auricchio F, da Veiga LB, Buffa A, Lovadina C, Reali A, Sangalli G (2007) A fully "locking-free" isogeometric approach for plane linear elasticity problems: a stream function formulation. Comput Methods Appl Mech Eng 197:160-172

Bazilevs Y, da Veiga LB, Cottrell JA, Hughes TJR, Sangalli G (2006) Isogeometric analysis: approximation, stability and error estimates for $h$-refined meshes. Math Models Methods Appl Sci 16(7):1031-1090

Bazilevs Y, Calo VM, Cottrell JA, Hughes TJR, Reali A, Scovazzi G (2007) Variational multiscale residual-based turbulence modeling for large eddy simulation of incompressible flows. Comput Methods Appl Mech Eng 197:173-201 
Bazilevs Y, Calo VM, Cottrell JA, Evans JA, Hughes TJR, Lipton S, Scott MA, Sederberg TW (2010) Isogeometric analysis using Tsplines. Comput Methods Appl Mech Eng 199:229-263

Bletzinger KU (1993) Extended method of moving asymptotes based on second order information. Struct Optim 5:175-183

Brach RM (1968) On the extremal fundamental frequencies of vibrating beams. Int J Solids Struct 4(7):661-666

Chang KH, Choi KK (1992) A geometry-based parameterization method for shape design of elastic solids. Mech Struct Mach 20(2):215-252

Cho S, Ha SH (2009) Isogeometric shape design optimization: exact geometry and enhanced sensitivity. Struct Multidisc Optim 38(1):53-70

Choi G, Kwak BM (2009) Application of isogeometric analysis to shape optimal design of thermal problems. In: Proceedings of the 8th world congress on structural and multidisciplinary optimization

Cottrell JA, Reali A, Bazilevs Y, Hughes TJR (2006) Isogeometric analysis of structural vibrations. Comput Methods Appl Mech Eng 195:5257-5296

Cottrell JA, Hughes TJR, Reali A (2007) Studies of refinement and continuity in isogeometric structural analysis. Comput Methods Appl Mech Eng 196:4160-4183

Cottrell JA, Hughes TJR, Bazilevs Y (2009) Isogeometric analysis: toward the integration of CAD and FEA. Wiley, Singapore

Echter R, Bischoff M (2009) Numerical efficiency, locking and unlocking of nurbs finite elements. Comput Methods Appl Mech Eng 199:374-382

Evans JA, Bazilevs Y, Babuška I, Hughes TJR (2009) n-Widths, sup-infs, and optimality ratios for the $k$-version of the isogeometric finite element method. Comput Methods Appl Mech Eng 198(21-26):1726-1741

Gajewski A, Piekarski Z (1994) Optimal structural design of a vibrating beam with varying cross-section. Struct Optim 7:112-116

Ha SH, Cho S, Choi KK (2008) Shape design optimization using isogeometric approach. In: Proceedings of the 12th AIAA/ISSMO multidisciplinary analysis and optimization conference

Haftka RT, Grandhi RV (1986) Structural shape optimization-a survey. Comput Methods Appl Mech Eng 57:91-106

Haftka RT, Gürdal Z (1992) Elements of structural optimization. Kluwer, Dordrecht

Hassani B, Khanzadi M, Tavakkoli SM, Moghadam NZ (2009) Isogeometric shape optimization of three dimensional problems. In: Proceedings of the 8th world congress on structural and multidisciplinary optimization

Hughes TJR, Cottrell JA, Bazilevs Y (2005) Isogeometric analysis: CAD, finite elements, NURBS, exact geometry and mesh refinement. Comput Methods Appl Mech Eng 194:4135-4195

Hughes TJR, Reali A, Sangalli G (2008) Duality and unified analysis of discrete approximations in structural dynamics and wave propagation: comparison of $p$-method finite elements with $k$-method NURBS. Comput Methods Appl Mech Eng 197(49-50):41044124

Hughes TJR, Reali A, Sangalli G (2010) Efficient quadrature for NURBS-based isogeometric analysis. Comput Methods Appl Mech Eng 199:301-313
Kamat MP, Simitses GJ (1973) Optimal beam frequencies by the finite element displacement method. Int J Solids Struct 9:415429

Karihaloo BL, Niordson FI (1973) Optimal design of vibrating cantilevers. J Optim Theory Appl 11(6):638-654

Katsikadelis JT, Tsiatas GC (2006) Regulating the vibratory motion of beams using shape optimization. J Sound Vib 292:390-401

Nagy AP, Abdalla MM, Gürdal Z (2010) Isogeometric sizing and shape optimisation of beam structures. Comput Methods Appl Mech Eng 199:1216-1230

Niordson FI (1965) On the optimal design of a vibrating beam. Q Appl Math 23(1):47-53

Olhoff N (1976) Optimization of vibrating beams with respect to higher order natural frequencies. J Struct Mech 4(1):87-122

Olhoff N (1977) Maximizing higher order eigenfrequencies of beams with constraints on the design geometry. J Struct Mech 5(2): $107-134$

Olhoff N (1989) Multicriterion structural optimization via bound formulation and mathematical programming. Struct Optim 1:11-17

Olhoff N, Plaut RH (1983) Bimodal optimization of vibrating shallow arches. Int J Solids Struct 19(6):553-570

Piegl L, Tiller W (1995) The NURBS book-monographs in visual communication, 2nd edn. Springer, Heidelberg

Plaut RH, Olhoff N (1983) Optimal forms of shallow arches with respect to vibration and stability. J Struct Mech 11(1):81-100

Rozvany GIN (1989) Structural design via optimality criteria. Kluwer, Dordrecht

Shen J, Yoon D (2003) A new scheme for efficient and direct shape optimization of complex structures represented by polygonal meshes. Int J Numer Methods Eng 53:2201-2223

Simo JC (1984) A finite strain beam formulation. The threedimensional dynamic problem. Part I. Comput Methods Appl Mech Eng 49:55-70

Svanberg K (2002) A class of globally convergent optimization methods based on conservative complex separable approximations. SIAM J Optim 12(2):555-573

Svanberg K (2007) MMA and GCMMA. Tech. rep.

Szabó B, Düster A, Rank E (2004) The $p$-version of the finite element method. In: Encyclopedia of computational mechanics, vol 1fundamentals. Wiley, New York

Takewaki I (1996) Optimal frequency design of tower structures via an approximation concept. Comput Struct 58(3):445-452

van Keulen F, Haftka RT, Kim NH (2005) Review of options for structural design sensitivity analysis. Part 1: linear systems. Comput Methods Appl Mech Eng 194:3213-3243

Wall WA, Frenzel MA, Cyron C (2008) Isogeometric structural shape optimization. Comput Methods Appl Mech Eng 197:2976-2988

Wesseling P (2001) Principles of computational fluid dynamics. Springer, Heidelberg

Zhang WH, Fleury C, Duysinx P (1996) A generalized method of moving asymptotes (GMMA) including equality constraints. Struct Optim 12:143-146

Zhou M, Pagaldipti N, Thomas HL, Shyy YK (2004) An integrated approach to topology, sizing and shape optimization. Struct Multidisc Optim 26:308-317 